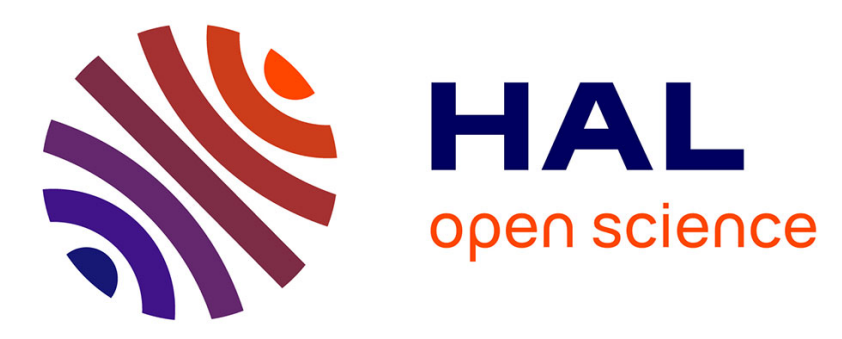

\title{
U-shaped response Unifies views on temperature dependency of stoichiometric requirements
}

Thomas Ruiz, Apostolos-manuel Koussoroplis, Michael Danger, Jean-pierre Aguer, Nicole Morel-desrosiers, Alexandre Bec

\section{- To cite this version:}

Thomas Ruiz, Apostolos-manuel Koussoroplis, Michael Danger, Jean-pierre Aguer, Nicole Moreldesrosiers, et al.. U-shaped response Unifies views on temperature dependency of stoichiometric requirements. Ecology Letters, 2020, 23 (5), pp.860-869. 10.1111/ele.13493 . hal-03227608

\section{HAL Id: hal-03227608 \\ https://hal.science/hal-03227608}

Submitted on 29 Oct 2021

HAL is a multi-disciplinary open access archive for the deposit and dissemination of scientific research documents, whether they are published or not. The documents may come from teaching and research institutions in France or abroad, or from public or private research centers.
L'archive ouverte pluridisciplinaire HAL, est destinée au dépôt et à la diffusion de documents scientifiques de niveau recherche, publiés ou non, émanant des établissements d'enseignement et de recherche français ou étrangers, des laboratoires publics ou privés. 


\section{U-shaped response Unifies views on temperature dependency of stoichiometric requirements}

\section{Thomas Ruiz, ${ }^{1 *}$ iD Apostolos- Manuel Koussoroplis, ${ }^{1}$ Michael Danger, ${ }^{2}$ (iD Jean-Pierre Aguer, ${ }^{1}$ Nicole Morel-Desrosiers ${ }^{1}$ and Alexandre Bec ${ }^{1}$}

The peer review history for this article is available at $h$ ttps://publons.c om/publon/10.1111/ele.13493

\begin{abstract}
Temperature and nutrient availability, which are major drivers of consumer performance, are dramatically affected by global change. To date, there is no consensus on whether warming increases or decreases consumer needs for dietary carbon $(\mathrm{C})$ relatively to phosphorus $(\mathrm{P})$, thus hindering predictions of secondary production responses to global change. Here, we investigate how the dietary C:P ratio optimising consumer growth $\left(T E R_{C: P}\right.$ : Threshold Elemental Ratio) changes along temperature gradients by combining a temperature-dependent $T E R_{C: P}$ model with growth experiments on Daphnia magna. Both lines of evidence show that the $T E R_{C: P}$ response to temperature is U-shaped. This shape indicates that consumer nutrient requirements can both increase or decrease with increasing temperature, thus reconciling previous contradictive observations into a common framework. This unified framework improves our capacity to forecast the combined effects of nutrient cycle and climatic alterations on invertebrate production.
\end{abstract}

\section{Keywords}

Daphnia, ectotherm, global change, nutrient, stoichiometry, temperature, TER.

\section{INTRODUCTION}

In the last decades, temperature of lake surface water increased on average by $0.34{ }^{\circ} \mathrm{C}$ decade $^{-1}$ (O'Reilly et al., 2015), a trend which may worsen regarding the speed at which global change occurs (Rogelj et al., 2012). In the same time, urbanisation and agricultural practices lead to significant alterations of elemental cycles (especially $\mathrm{C}, \mathrm{N}$ and $\mathrm{P}$ ) and nutrient availability in the biosphere (Falkowski et al., 2000). These two factors are important drivers of ectotherm herbivore physiology, temperature regulating the rate of all biological process occurring in the organism (Angilletta, 2006) and nutrient uptake fuelling physiological needs for growth or reproduction (Elser et al., 2003). The independent effects of temperature and nutrient availability on organisms has long been studied separately, however, in natural environments submitted to global change, these two factors change in parallel, affecting consumer performance interactively (Kingsolver et al., 2006). Hence, it is essential to consider any temperature change within its nutritional context to understand on-going and future changes in invertebrates' populations and their interactions with other trophic levels.

Several studies have explored the thermal sensitivity of consumer nutritional requirements, particularly focusing on $\mathrm{C}: \mathrm{N}$ : P stoichiometry (Cross et al., 2015) and two competing predictions emerge from this literature. The first emphasises the temperature sensitivity of individual metabolic rate (Brown et al., 2004) arguing that a higher metabolic activity resulting from higher temperature should increase the energetic requirements of the organism. As energy results from the oxidation of C-rich compounds, an increased temperature should therefore increase consumer $\mathrm{C}$ needs. This hypothesis is supported by experimental evidence both for terrestrial (Lee et al., 2015) or aquatic (Boersma et al., 2016; Malzahn et al., 2016) consumers which tend to select for $\mathrm{C}$-rich diets at higher temperatures. However, other authors claim that higher temperature also accelerates growth (Frazier et al., 2006). According to the growth rate hypothesis (Elser et al., 2003), an increased growth rate should raise nutrient demand $(\mathrm{N}$ and $\mathrm{P})$ to sustain increasing protein synthesis and DNA replication. This hypothesis is also supported by empirical evidence showing an increasing nutrient demand at higher temperatures both in terrestrial (Lemoine et al., 2013) or aquatic (Persson et al., 2011; Wojewodzic et al., 2011) consumers. To date, these apparently contradictive results remain a conundrum and the evolution of consumer stoichiometric requirements under warming scenarios a subject of vivid debate (Boersma et al., 2016; Winder et al., 2016). A recent opinion paper by Schmitz \& Rosenblatt (2017), however, contains an appealing solution to the above controversy. The authors propose four theoretical models for the interactive effects between temperature and predation stress on consumer stoichiometric requirements. One of their models predicts that both $\mathrm{C}$ and nutrient demand can increase with warming and that previous contradictive results could simply reflect different regions of the same underlying U-shaped response.

The models in Schmitz \& Rosenblatt (2017) are based on the threshold elemental ratio (TER), a well-established framework defining how consumer biomass production responds to the elemental composition of food (Frost et al., 2006). The TER is the ratio at which elements should be present in diet to maximise consumer growth and where the limitation of growth switches from one element to another. The most commonly studied TER are those relating carbon (C) to
${ }^{1}$ Université Clermont Auvergne, CNRS, LMGE, Clermont-Ferrand F-63000, France

\footnotetext{
${ }^{2}$ Université de Lorraine, CNRS, LIEC, Metz F-57000, France

*Correspondence: E-mail: thomas.ruiz@uca.fr
} 
phosphorus $(\mathrm{P})$ or nitrogen $(\mathrm{N})$ and they reflect the switching point from an energy-limited (i.e. C-limited) growth to a nutrient-limited growth. Deviations of food stoichiometry bellow or above the TER induce a dietary (C- or nutrient-) limitation for consumers (Frost et al., 2006) and lead to a unimodal response of growth to food stoichiometry also known as the "stoichiometric knife-edge" (Elser et al., 2016; Zhou \& Declerck, 2019). Besides the effects on consumer performance, the TER can explain consumer-driven $\mathrm{P}$ and $\mathrm{N}$ recycling in ecosystems (Cherif \& Loreau, 2013), population dynamics (Elser et al., 2000; Frost et al., 2006) and species interactions (Boersma et al., 2016). The main innovation in Schmitz \& Rosenblatt (2017) is that certain physiological processes underlying the TER such as mass-specific ingestion and respiration rate do not simply rise exponentially as usually assumed (Anderson et al., 2017) but rather exhibit a unimodal response to temperature (McMahon, 1969; Kibby, 1971; Yurista, 1999). Under this assumption, the TER of a consumer can exhibit a U-shaped response to temperature, an exciting prediction that remains to be tested experimentally.

The aim of our study is twofold. First, we extend the theoretical work of Schmitz \& Rosenblatt (2017) and thoroughly explore the conditions under which the U-shaped response of the TER emerges. Departing from a model parametrisation based on the freshwater cladoceran Daphnia magna, we analyse the robustness of the U-shape to variations in the thermal reaction norms of the physiological traits underlying the TER. Second, we experimentally determine how the TER of a Daphnia magna clone changes with temperature by measuring its somatic growth responses under a factorial combination of temperatures and dietary stoichiometry.

\section{MATERIAL AND METHOD}

\section{Model equations and analysis}

The $T E R_{C: n u t r i e n t}$ can be expressed as the product of nutrient use efficiency and the consumer's elemental composition:

$T E R_{C: n u t r i e n t}=\frac{A E_{\text {nutrient }}}{G G E c} * Q_{\frac{c}{\text { nutrient }}}$

where $Q_{C \text { :nutrient }}$ is the body composition of organisms, $A E_{\text {nutrient }}$ is the assimilation efficiency of a specific nutrient $(\mathrm{N}$ or P) and $G G E_{C}$ the gross growth efficiency of $C . G G E_{C}$ refers to the proportion of ingested $\mathrm{C}$ used for growth, formulated as:

$G G E_{C}=\frac{I_{C} * A E_{C}-R_{C}}{I_{C}}$

where $I_{C}$ is the $\mathrm{C}$ ingestion rate, $A E_{C}$ the $C$ assimilation efficiency and $R_{C}$ the $C$ respired.

In a comparable approach to Schmitz \& Rosenblatt (2017), we define the thermal reaction norms for each process underlying the TER. The thermal response of daphnia ingestion rate has been largely documented (McMahon, 1969; Kibby, 1971; Yurista, 1999) and is known to present a temperature optimum below and above which process rate decreases generating a hump-shaped thermal performance curve (TPC) (Angilletta, 2006). We modelled $I_{C}$ using a modified Gaussian as:
$I_{C}=I_{C M I N}+\Delta I_{C M A X} * e^{-b\left(T-T_{O P T}\right) 2}$

where $\triangle I_{C M A X}$ is the difference between minimum $\left(I_{C M I N}\right)$ and maximum ingestion rate. $T$ is temperature, $T_{O P T}$ the optimal temperature and $b$ a coefficient determining the decrease rate around $T_{O P T}$. Here, we estimated the shape of $\mathrm{C}$ ingestion TPC parameters $\left(b, I_{C M I N} / \triangle I_{C M A X}\right.$ ratio) by fitting eqn 3 to the measurements of Yurista et al. (1999) on the artic cladoceran Daphnia middendorfiana. However, to adjust the TPC to our temperate D. magna we replaced the thermal optimum $\left(T_{O P T}\right)$ to $22{ }^{\circ} \mathrm{C}$, the acclimation temperature of our clones (Fig. 1). We also adjusted the maximum ingestion rate $\left(I_{C M I N}+\Delta I_{C M A X}\right)$ to the measurement of Urabe \& Watanabe (1991) on temperate Daphnia species fed on Chlamydomonas reinhardtii, which is also in accordance with Darchambeau et al. (2003). However, as data on thermal dependence of $I_{C}$ are scarce for Daphnia magna (McMahon, 1969), we tested how the $T E R_{C: P}$ thermal reaction is influenced by curvature of the $I_{C}$ hump-shaped response by varying $b$ in the sensitivity analysis (Fig. 1a).

In Schmitz \& Rosenblatt (2017), respiration is modelled as a hump-shaped thermal reaction norm. Although this is valid, experimental evidence indicates that within the temperature range we used in our study, the metabolic rate of D. magna (i.e. respiration) rises exponentially. Hence, unlike Schmitz \& Rosenblatt (2017), we modelled $R_{c}$ as:

$R_{c}=a e^{d T}$

where $d$ is the scaling exponent and $a$ the proportionality constant. We measured D. magna metabolic rate (see method below) to confirm the exponential rise. Fitting our data to eqn 4 results in a scaling parameter $d=0.035$ (Fig. S1). We also tested how the predicted $T E R_{C: P}$ reaction norm is affected by variations in the scaling exponent $d$ ranging from 0.01 to 0.07 (Fig. 1b).

Carbon and nutrient assimilation efficiency $(A E)$ are typically assumed constant across temperatures in zooplankton TER models (Frost et al., 2006; Anderson et al., 2017) and experimental evidence seems to support this assumption (Yurista, 1999). Using previously reported values for Daphnia as reference we modelled $A E_{C}$ and $A E_{P}$ constant with temperature $\left(A E_{C} \sim 0.5\right.$ (Urabe \& Watanabe, 1991; Yurista, 1999) and $A E_{P} \sim 0.8$ (Anderson \& Hessen, 2005; Frost et al., 2006)). As levels of $A E_{C}$ and $A E_{P}$ reported in literature do not constitute a clear consensus, we tested how the $T E R_{C: P}$ thermal reaction norm responds to increases and decreases in $A E_{C}$ and $A E_{P}$ levels around these reported values (Fig. 1c and d). See Supplementary material for a model with $A E$ thermal reaction norms (Fig. S2).

We analysed the elemental content of our Daphnia clone (see method below) and showed no significant differences in body C:P ratio (Fig. S3) across dietary and temperature treatments, with an average value of 95 . Such homeostatic regulation is not uncommon in daphnids fed stoichiometrically imbalanced. However, some studies on other D. magna clones and copepods suggested that body C:P can either increase (McFeeters \& Frost, 2011) or decrease (Mathews et al., 2018) with increasing temperatures. To test all these scenarios (constant, increasing and decreasing $\left.Q_{C: P}\right)$, we define $Q_{C: P}$ as: 
(a)

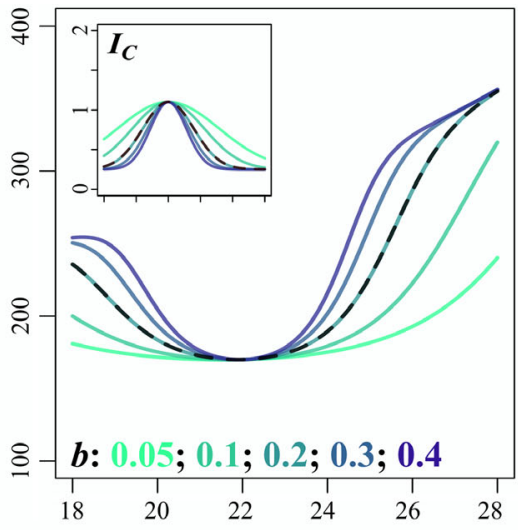

(b)

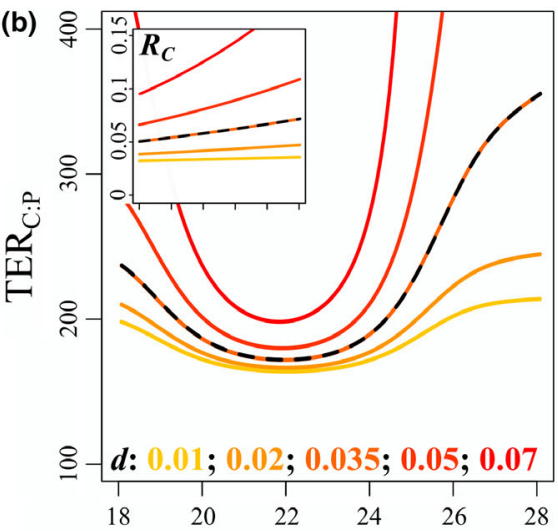

(c)

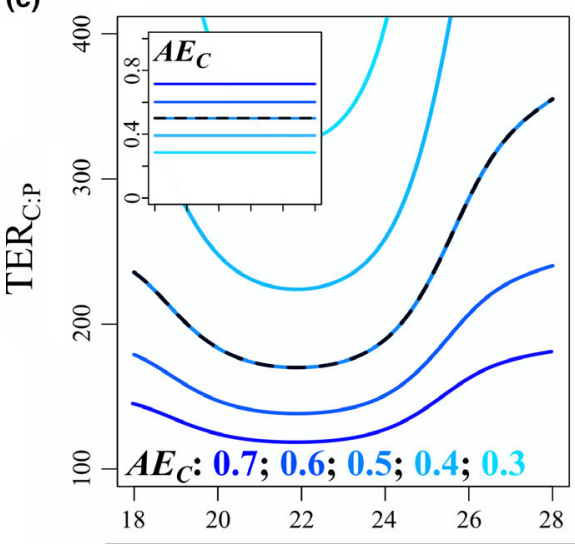

(d)

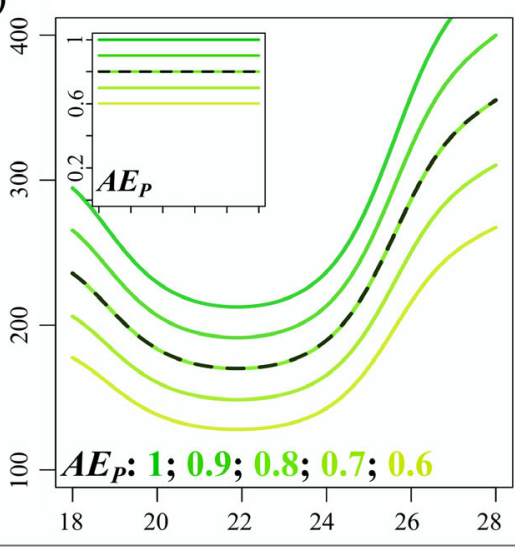

(e)

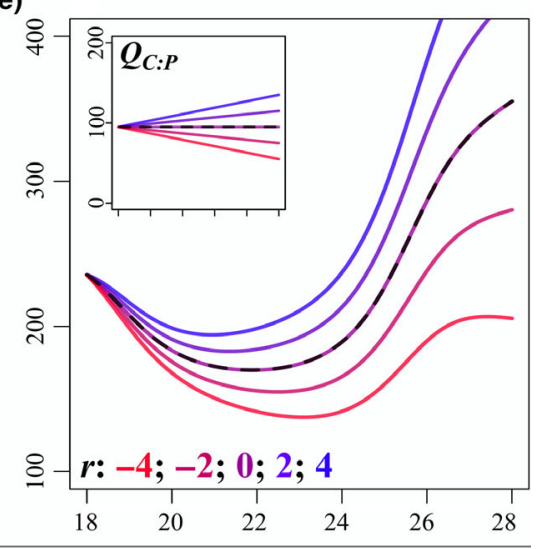

Temperature $\left({ }^{\circ} \mathrm{C}\right)$

Figure 1 Effect of parameter values on the thermal reaction norm of TER. Each panel is associated with the variation in one specific parameter constraining: (a) The concavity (parameter $b$ ) of the carbon ingestion rate reaction norm $I_{c}$, (b) the temperature scaling (parameter $d$ ) of carbon respiration $R_{C}$, (c) the level of carbon assimilation efficiency $A E c$, (d) the level of phosphorus assimilation efficiency $A E_{P}$ and (e) the slope (parameter $r$ ) of consumer's body C:P response to temperature. Each parameter is varied individually while the others are kept constant at the values for $D$. magna (see Table 1). Upper left inserts present the temperature responses of physiological process associated with parameter variations. The dashed black line is the model prediction for D. magna. See Table 1 for details on the selected parameter ranges.

$Q_{C: P}(T)=C P_{0}+\left(r *\left(T-T_{M I N}\right)\right)$

where $C P_{0}$ and $r$, respectively, define the intercept and slope of the $Q_{C: P}$ versus temperature curve and $T_{M I N}$ the minimal temperature observed (Fig. 1e). By setting $r$ to $0, Q_{C: P}$

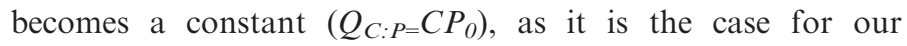
D. magna clone.

To test the robustness of our predictions, we calculated the $T E R$ thermal reaction norm (from Eq.1), for 1000 random combinations of parameter values (parameters $b, d, A E_{C}$, $\left.A E_{p}, r\right)$ sampled from uniform distributions bound within the biologically relevant ranges defined in Table 1. For each obtained TER reaction norm, we calculated the average slope (at $0.5{ }^{\circ} \mathrm{C}$ intervals) below and above optimal temperature (set at $22{ }^{\circ} \mathrm{C}$ ) to describe the shape of $T E R_{C: P}$ thermal reaction. Only slopes above $5 \%$ were considered significant. When both slopes are positive or negative or when one of them is zero, we considered the $T E R_{C: P}$ thermal reaction as monotonous. A U-shape (soft or strong) was assigned when the slope is initially negative and then positive. A strong-U $T E R_{C: P}$ slope was assigned when both slopes are above $10 \%$ (Fig. 2).

\section{Daphnia maintenance}

We tested our model predictions using a clonal line of Daphnia magna to exclude genotypic response variations. The stock cultures of daphnids were kept in Volvic water ${ }^{\circ}$ at $22{ }^{\circ} \mathrm{C}$ on a 14:10 h day:night cycle and fed with Chlamydomonas reinhardtii (C:P 250 at $3 \mathrm{mgC} \mathrm{L}^{-1}$ ) for more than three generations.

\section{Preparation of food suspensions}

Chlamydomonas reinhardtii was used as food in all experiments. We used three freeze-dried stock cultures with respective molar C:P ratio of 95 (high P), 331 (intermediate P) and 1014 (low P) previously prepared (See supplementary material for details). Food suspensions were prepared daily by mixing freeze-dried algae stocks diluted in Volvic ${ }^{\odot}$ water (Table. S1). Despite a slight reduction in individual performance, freezedried algae were shown to be adequate food for D. magna for several generations (Naylor et al., 1993). The $\mathrm{P}$ content of algae has no significant influence on their fatty acids composition (Table. S2) and the rehydration of freeze-dried algae had no effect on their elemental composition. 
Table 1 Modelled thermal reaction norms of the physiological processes underlying the TER model in eqn 1 and associated parameters. Tested parameter ranges (see Fig. 1) are given in parentheses, values in bold are the values used for Daphnia magna. ${ }^{1}$ (Yurista, 1999); ${ }^{2}$ (Urabe \& Watanabe, 1991); ${ }^{3}$ (Anderson \& Hessen, 2005); ${ }^{4}$ (Frost et al., 2006).

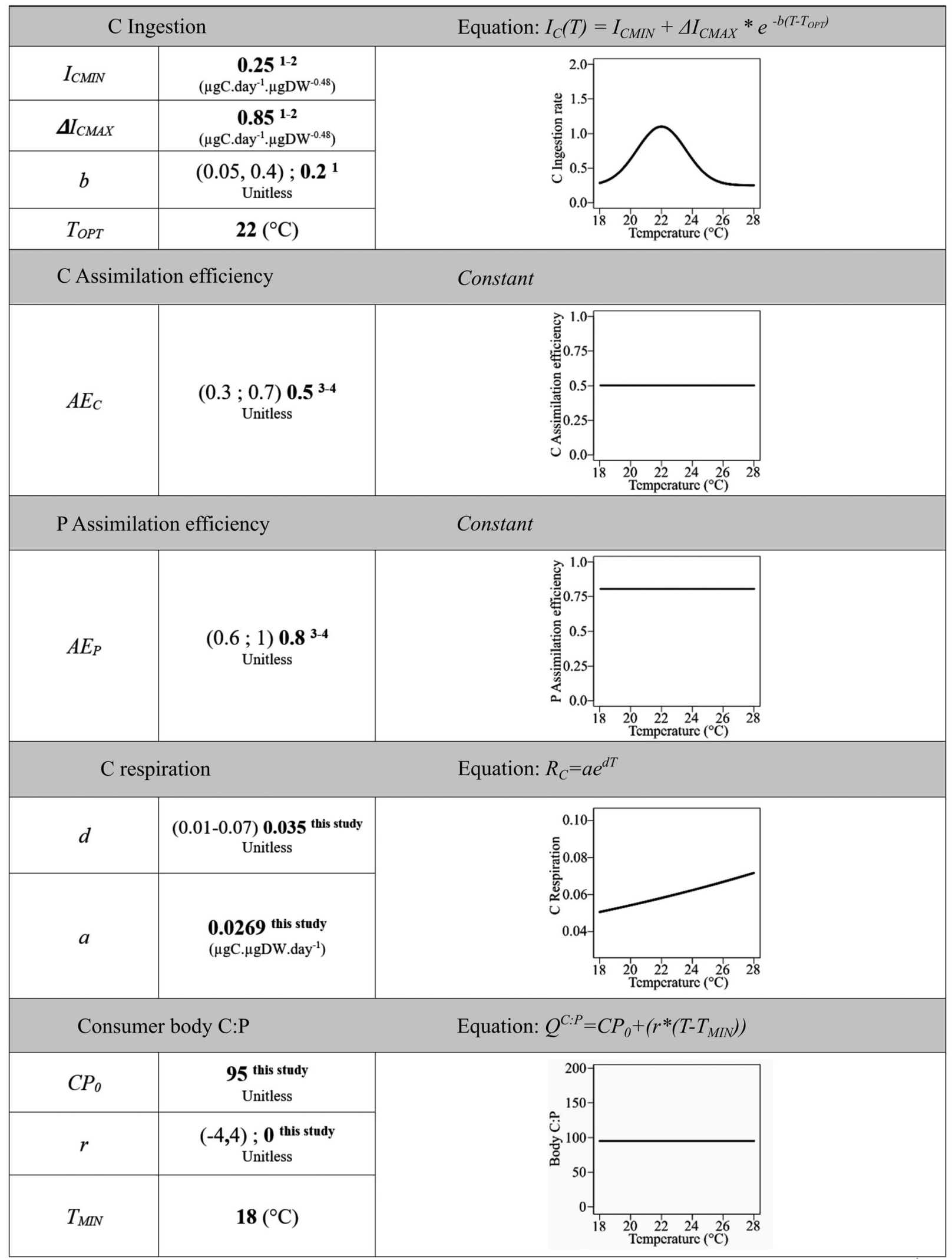




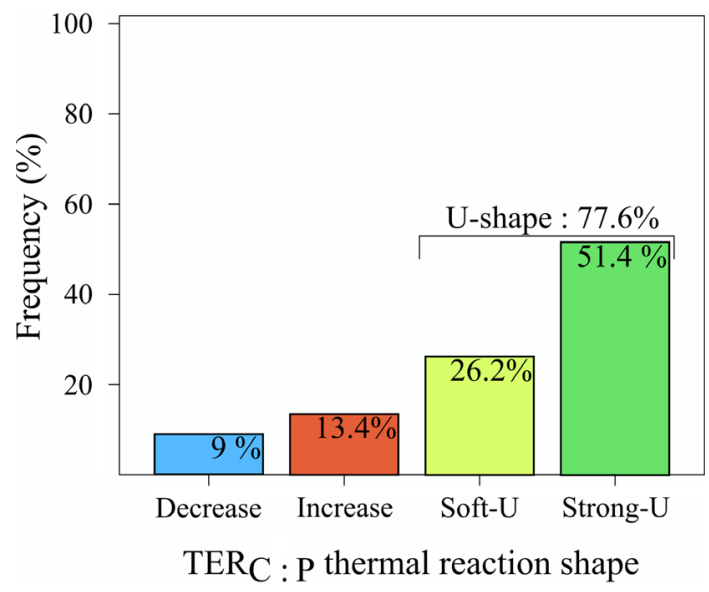

Figure 2 Frequency of occurrence of $T E R_{C: P}$ thermal reaction norms across 1000 random model parameter combinations. Parameter values are sampled within the ranges given in Table 1 assuming uniform distributions. A U-shape is considered "strong" when both lines have a slope above 10\%. Decrease and Increase indicate a monotonically increasing or regressing reaction norms respectively. Error on slopes estimates is set at 5\%. See methods for details. See Figure S4 for detailed parameter distributions of each pattern.

\section{Experimental procedure}

We used a gradient experimental design to capture the nonlinearity of D. magna growth rate response to diet stoichiometry and temperature (Kreyling et al., 2018). Daphnids were grown on a full factorial combination of six stoichiometric treatments (with C:P ratios of 95-172-250-331-553-1014, obtained by mixing the freeze-dried stock cultures) at six different temperatures (18-20-22-24-26 and $\left.28^{\circ} \mathrm{C}\right)$. For technical reasons, however, growth experiments were performed independently for each temperature using the same clonal line and a strictly identical protocol. At the beginning of the experiments, 8-hold neonates obtained from the third or fourth clutch were randomly distributed in two replicate glass jars $(250 \mathrm{~mL}, 12$ ind/jar) per dietary treatments. The culture medium composed of Volvic $\subset$ water with ad libitum food $\left(3 \mathrm{mgC} \mathrm{L}^{-1}\right)$ was renewed daily to maintain food quantity above the saturating level during all the experiment. Individuals were raised until they reached maturity, which took from 5 to 9 days depending on temperature. They were then pooled by jar, dried $48 \mathrm{~h}$ at $60^{\circ} \mathrm{C}$, weighed (Sartorius-ME36S; Germany; precision:0.001 $\mathrm{mg}$ ) and stored until elemental analysis. The average growth rate in each jar was calculated as follows:

Growth rate $=\frac{\ln (W t)-\ln (W 0)}{t}$

where $W_{t}$ is the average weight $(\mu \mathrm{g})$ at age of maturity $t$ (days) and $W_{0}$ the average weight $(\mu \mathrm{g})$ of neonates. The growth rate thus calculated refers to the juvenile growth rate which has been shown to represent the best proxy for population increase rate (Lampert \& Trubetskova, 1996). After measuring the mean weight, pools of dried daphnids were divided into subgroups to measure their carbon and phosphorus content. In parallel, a subset of mature daphnids from the C:P 250 treatment was used to estimate their metabolic rate. As variation in metabolic rate (a.k.a. respiratory rate) with diet is not included in TER model, we performed this measurement on a single dietary treatment which is the diet that daphnids used to consume. The mass-specific metabolic rates obtained by microcalorimetry (Ruiz et al. 2018) were plotted against temperature to determine the temperature dependence of metabolism (Fig. S2).

\section{Elemental and biochemical analyses}

Elemental composition of algae and daphnids was performed in a similar way. Carbon was determined using a $\mathrm{CHN}$ analyser (NA-2100-Protein, ThermoQuest-CE Instruments, Italy) and phosphorus content was estimated using a colorimetric determination based on potassium persulfate method (Ruiz et al., 2018). Fatty acid of algae was extracted using chloroform/methanol method and then converted into fatty acid methyl esters by acid-catalysed transesterification (Denoux et al., 2017). The fatty acid methyl esters were analysed by gas chromatography (6850-Network-GC, Agilent-technologies, USA).

\section{Data analyses}

To model the response surface of $D$. magna juvenile growth rate to experimental gradients of temperature and dietary C:P, we fitted a Generalised Additive Model (GAM) to the experimental growth rate data. The model of growth rate was constructed as follows:

$G R_{T, C: P}=f\left(x_{T}\right)+f\left(x_{C: P}\right)+t i\left(x_{T}, x_{C: P}\right)$

where $f\left(x_{T}\right)$ and $f\left(x_{C: P}\right)$ are the smooth terms for temperature and diet stoichiometry, whereas $t i\left(x_{T}, x_{C: P}\right)$ is the tensor interaction smooth term. The model was fitted to the data using the mgev R package (Wood, 2001) and "Restricted Maximum Likelihood" REML method.

To assess the shape of the $T E R_{C: P}$ thermal reaction norm, we first estimated the $T E R_{C: P}$ at each experimental temperature separately. For each temperature, we fitted the following modified Gaussian function to the growth rate versus C:P data:

$G R_{T}=a+b * e^{-E * \frac{x_{C \cdot P} \cdot-c^{2}}{d^{2}}}$

where $x_{C: P}$ is the resources C:P ratio, $a$ the minimum growth rate, $b$ is the height of the curve, $c$ is the estimated $T E R_{C: P}$ (i.e. curve's optimum), $d$ the curve breadth and $E$ a scaling parameter. The various parameters including $c\left(T E R_{C: P}\right)$ were estimated by nonlinear least squares regression. The confidence intervals around the $T E R_{C: P}$ were estimated by nonparametric bootstrapping (Efron \& Tibshirani, 1986). At each temperature the dataset was resampled 1000 times and the nonlinear regression procedure and $T E R_{C: P}$ estimation reiterated. The obtained estimates are strongly robust against the number of bootstrap iterations (Table S3) and were used to calculate the mean $T E R_{C: P}$ and the $95 \%$ confidence intervals $\left(\mathrm{CI}_{95 \%}\right)$. A significant difference between $T E R_{C: P}$ estimates at each temperature was concluded in the absence of overlap between their $\mathrm{CI}_{95 \%}$. 
Finally, to determine whether the $T E R_{C: P}$ reaction norm has a true U-shape we used the two-line test (Simonsohn, 2018) on the bootstrapped data. Briefly, the two-line test is used to statistically confirm or infirm a U-shape in data series. The test consists of estimating two regression lines, for "low" and "high" values of $\mathrm{x}$ separated at a specific breakpoint. The test confirms a U-shape if the two lines have opposite and individually significant slopes. All analyses were performed using the software R v.3.6.1 (R-Core Team 2019) with the alpha error set at 0.05 .

\section{RESULTS}

\section{Model}

When parameterised for Daphnia (Table 1), the model predicts that the $T E R_{C: P}$ thermal reaction norm is U-shaped, decreasing between 18 and $22{ }^{\circ} \mathrm{C}$ and then increasing up to $28^{\circ} \mathrm{C}$ (Fig. 1, dashed line). The TER C:P is thus minimised at $22^{\circ} \mathrm{C}$ which constitutes the threshold minimising temperature (TMT). Among the model parameters, $b$, which controls the concavity of the $\mathrm{C}$ ingestion rate thermal reaction norm $\left(I_{c}\right)$ has a strong effect on the convexity of $T E R_{C: P}$ thermal response (Fig. 1a). Similarly, the temperature scaling exponent of respiration $\left(R_{c}\right), d$, has a strong effect on the $T E R_{C: P}$ (Fig. 1b). The lowest $d$ value used here $(0.01)$, which corresponds to a $\mathrm{Q}_{10}=1.2$ for $R_{c}$, yields a nearly flat $T E R_{C: P}$ reaction. Increasing the temperature scaling of $R_{c}$ up to a $\mathrm{Q}_{10}=2$ (parameter $d=0.07$ ) strongly increases the convexity of the $T E R_{C: P}$ thermal reaction norm and shifts the minimal value of $T E R_{C: P}$ toward higher C:P ratios. The level of $\mathrm{C}$ assimilation efficiency $\left(A E_{C}\right)$ also constrains both the level and the convexity of the $T E R_{C: P}$ thermal reaction norm. Reducing $A E_{C}$ from 0.7 to 0.3 strongly increases the level and convexity of the reaction norm (Fig. 1c). The assimilation efficiency of $\mathrm{P}\left(A E_{P}\right)$ also modulates the thermal reaction of $T E R_{C: P}$ in a lesser extent. Increasing $A E_{P}$ values from 0.6 to 1 only shifts $T E R_{C: P}$ thermal reaction norm to higher $\mathrm{C}: \mathrm{P}$ values without altering its convexity (Fig. 1d). The scaling of consumer body composition $\left(Q_{C: P}\right)$ with temperature (defined by parameter $r$ ) only affects $T E R_{C: P}$ at higher temperatures. Increasing $Q_{C: P}$ with temperature (parameter $r=4$ ) leads to a rapid increase in $T E R_{C: P}$ at higher temperatures. Oppositely, decreasing $Q_{C \text { : }}$ ${ }_{P}$ at high temperature $(r=-4)$ tends to limit $T E R_{C: P}$ to lower values, slightly reducing the convexity of its thermal response without flattening the U-shape (Fig. 1e).

The sensitivity analyses of the model show that across a large number $(n=1000)$ of random parameter combinations (selected within biologically relevant ranges, see Table 1 ), the Ushape $T E R_{C: P}$ thermal reaction norm is predominant with a frequency of occurrence of $77.6 \%$ (Fig. 2). More precisely, softly convex U-shapes occur in $26.2 \%$ of observations and strongly convex U-shapes in $51.4 \%$. The remainder of simulation outcomes are monotonic increases $(13.4 \%)$ or decreases $(9 \%)$ of $T E R_{C: P}$ with temperature. These monotonic thermal reactions of the $T E R_{C: P}$ only emerge when $I_{C}$ thermal response is the less concave or when $R_{C}$ thermal reaction is flattened. In such situations, it is the thermal reaction norm of $Q_{C: P}$ that dictates the direction of the $T E R_{C: P}$ thermal response (Fig. S4).

\section{Experiments}

The surface response of daphnids growth across the factorial combination of dietary treatment and temperature is given in Fig. 3. The GAM used to generate the surface fit well with our data explaining $97.9 \%$ of total variance (Figs S5 and S6). The GAM also indicates a significant effect of the temperature and dietary C:P interaction on D. magna growth $(n=72 ; F=15.9$; $P<0.001)$. The response of growth to temperature is typically unimodal for diet C:P ratios ranging from 95 to 331, with an optimum ranging from 0.36 at $22{ }^{\circ} \mathrm{C}$ to 0.39 at $24{ }^{\circ} \mathrm{C}$, respectively (Fig. 3, Fig. S4). For C:P ratio above 500, the response of growth appears to be almost flat. Growth rate responded unimodally to diet at all temperatures (Fig. 3, Fig. S4) but the $T E R_{C: P}$ (i.e. the peak of the unimodal response) changed across temperatures forming a U-shape with a minimum value at $22{ }^{\circ} \mathrm{C}$. The $T E R_{C: P}$ decreased from $338.4\left(\mathrm{CI}_{95 \%}[326.9\right.$, $349.9])$ at $18{ }^{\circ} \mathrm{C}$ down to $193.4\left(\mathrm{CI}_{95 \%}[176.6,210.2]\right)$ at $22{ }^{\circ} \mathrm{C}$. The latter can be considered as our Daphnia clone's TMT as it represents the lowest $T E R_{C: P}$ observed in our thermal range. Further temperature increases above this TMT lead to an increase in the $T E R_{C: P}$ up to $414\left(\mathrm{CI}_{95 \%}[370.8,457.2]\right)$ at $28^{\circ} \mathrm{C}$ (Fig. 3). The two-line test of Simonsohn (2018) confirmed that $T E R_{C \cdot P}$ significantly decreases between 18 and $22{ }^{\circ} \mathrm{C}(P<0.0001)$, reaches a breakpoint at $22{ }^{\circ} \mathrm{C}$ after which $T E R_{C: P}$ significantly increases up to $28{ }^{\circ} \mathrm{C} \quad(P<0.0001)$ (Fig. 3), thus generating a significant U-shape.

\section{DISCUSSION}

Both our model and growth experiments showed that the $T E R_{C: P}$ of $D$. magna responds non-monotonically to temperature generating a "U-shaped" thermal reaction norm. The TER $C: P$ decreases between 18 and $22{ }^{\circ} \mathrm{C}$ indicating a rising demand in $\mathrm{P}$ with rising temperature. At $22{ }^{\circ} \mathrm{C}$, the $T E R_{C: P}$ reaches its

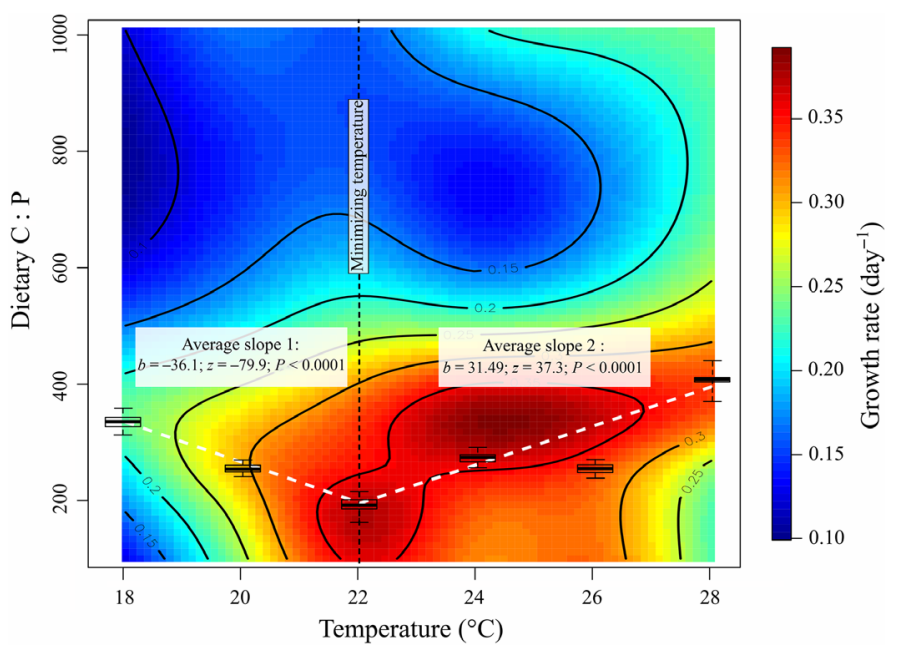

Figure 3 Generalised Additive Model (GAM; $n=72$, see also Fig. S3) of D. magna growth rate exposed to a factorial combination of dietary molar C:P ratio and temperature. The boxplots at each temperature are the bootstrapped $T E R_{C: P}$ mean values and confidence intervals $(n=1000)$. The shape of $T E R_{C: P}$ thermal reaction norm (white line) has been assessed with the two-line test (Simonsohn, 2018). Decreasing and increasing sections are both significant $(P<0.0001)$ 
minimal value, the Threshold Minimising Temperature (TMT) where the dietary $\mathrm{C}$ requirements relative to those of $\mathrm{P}$ are minimised. Above the TMT, and at least up to $28^{\circ} \mathrm{C}$, the $T E R_{C: P}$ increases again indicating a rising demand in dietary C. Consequently, for the same $D$. magna clone, both temperature-driven decrease and increase in the TER are possible, depending on the temperature range within which observations are performed. Provided that this $T E R_{C: P} \mathrm{U}$-shaped reaction norm can be generalised across consumers, our results suggest that previous apparent contradictory predictions of ectotherm consumers requirements in a warming context (Persson et al., 2011; Wojewodzic et al., 2011; Boersma et al., 2016) could be reconciled within a single framework.

Our model differs from those based on MTE that often assume that consumption is a monotonous function of temperature that has the same or higher $\mathrm{Q}_{10}$ than metabolism (Anderson et al., 2017). Such assumptions can only be valid within the lower part of consumer's thermal tolerance range. All else kept equal in our model, such assumptions will yield a monotonously decreasing $T E R_{C: P}$ thermal reaction norm. On the other hand, focusing only on the upper part of the consumers' thermal tolerance range where metabolism increases faster while consumption decreases will yield a monotonously increasing $T E R_{C: P}$ thermal reaction norms.

However, understanding ectotherm consumers' responses to climate change requires considering their full thermal tolerance range (Kingsolver \& Buckley, 2017). Within this range, C ingestion $\left(I_{c}\right)$ and respiration rates $\left(R_{c}\right)$ respond differently to temperature, both in terms of shape and scaling. It is these differential responses of $I_{c}$ and $R_{c}$ that generate the U-shaped TER reaction norm in our model. In agreement with empirical data on Daphnia (McMahon, 1969; Kibby, 1971; Yurista, 1999) and numerous other ectotherms (Dell et al., 2011; Lemoine \& Burkepile, 2012), we assume that within the full thermal tolerance range, the reaction norms of $I_{c}$ and $R_{c}$ are unimodal and exponential respectively. At the lower temperature range of the modelled $I_{c}$ reaction norm, ingestion rate rises faster than metabolic rate does (Yurista, 1999; Lemoine \& Burkepile, 2012). Under these circumstances and given a constant $\mathrm{C}$ assimilation efficiency $\left(A E_{C}\right)$, higher temperatures increase $\mathrm{C}$ gross growth efficiency $\left(G G E_{C}\right.$; eqn 2). The biological interpretation is that at higher body temperatures, the consumer "perceives" the food as being richer in C relative to $\mathrm{P}$. This manifests as a decrease in the $T E R_{C: P}$ with temperature until it reaches the TMT. In the model, the TMT indicates the tipping point where $R_{c}$ begins to rise faster than $I_{c}$. From this point, the $G G E_{C}$ starts decreasing with temperature and the consumer "perceives" the food as being increasingly "poorer" in $\mathrm{C}$ relative to $\mathrm{P}$ as temperature rises. Consequently, above the TMT, the $T E R_{C: P}$ rises with temperature. Hence, when considering a sufficiently wide thermal tolerance range, the $T E R_{C: P}$ exhibits a $\mathrm{U}$-shaped response to temperature.

The ingestion and respiration rates might not be the only model components affected by temperature. Although much less consensual, there is evidence that the assimilation efficiencies for $\mathrm{C}$ and $\mathrm{P}\left(A E_{C}\right.$ and $\left.\mathrm{AE}_{P}\right)$ as well as the consumer's body stoichiometry $\left(Q_{C: P}\right)$ may also respond to temperature (Bobka et al., 1981; Tsai et al., 2008; Prater et al., 2018). Depending on whether they increase or decrease with temperature, strong thermal responses could either "flatten" the U-shape, or one of its sides thereby yielding a quasi-monotonous $T E R_{C: P}$ thermal reaction norms. For example, unlike our D. magna clone, Prater et al (2018) found that the body stoichiometry of D. magna in two lakes tends to decrease with increasing temperature. In the model, such a decrease tends to flatten the rising part of the $T E R_{C: P}$ reaction norm. However, the range of $Q_{C: P}$ in Prater et al. (2018)(from 100 at $15{ }^{\circ} \mathrm{C}$ to 65 at $25^{\circ} \mathrm{C}$ ) is not enough to cause a monotonous decrease in $T E R_{C: P}$ with temperature in the model (Fig. 1e).

More generally, based on the model parameter ranges from literature, the simulation shows that $77.6 \%$ of random parameter combinations result in a U-shaped $T E R_{C: P}$ thermal reaction norm with $51.4 \%$ producing strongly convex U-shapes. This result suggests that U-shaped TER thermal reaction norms might be quite common among ectotherm consumers. Obviously this is a rough estimate and it needs be considered with caution. First, given the scarcity of data in literature, we assumed a uniform distribution for all parameter values. Improving the estimates of parameters distribution may potentially lead to different predictions but this is beyond the scope of this study. Second, parameter combinations in our simulation are random, and some of them might be biologically aberrant. For example, we could expect that in nature, higher ingestion rates are systematically associated with higher respiration rates in agreement with the increased intake hypothesis ((Burton et al., 2011) and lower $Q_{C: P}$ in agreement with growth rate hypothesis (Elser et al., 2003). Keeping only the biologically relevant combinations could alter the predicted frequency of occurrence of a U-shaped $T E R_{C: P}$.

A fundamental challenge in experimental ecology is to capture nonlinearities of ecological responses to environmental drivers. Our results underscore the importance of carefully selecting the temperature range and increments in any experimental assessment of temperature-dietary stoichiometry interaction on ectotherm consumers. In the case of strongly convex $T E R_{C: P}$ responses, large temperature increments $\left(>4{ }^{\circ} \mathrm{C}\right)$ might miss the U-shape and thus yield misleading conclusions. From an ecological perspective, strongly convex $T E R_{C: P}$ responses imply that small changes in temperature can drastically change consumer nutritional requirements. A diet of a given stoichiometry can shift towards one or the other side of $T E R_{C: P}$ within a range of few degrees Celsius. In agreement with the "knife-edge" theory (Elser et al., 2016), our experimental data show that both sides of the $T E R_{C: P}$ imply physiological costs which manifest as decrease in growth rate. Hence, consumers with strongly convex $T E R_{C: P}$ responses to temperature might be more susceptible to thermally induced changes in their dietary preferences (Boersma et al., 2016). Following the same reasoning, such consumers might also be more prone to behavioural thermoregulation to optimise nutritional outcomes (Coggan et al., 2011; Clissold et al., 2013). Furthermore, the U-shape of the $T E R_{C: P}$ thermal reaction norm implies that, for a given resource $C: P$, climate change-induced nutritional limitations could differ at the borders of the species geographical range. At the lower latitudinal border where the species is at the "warmer" part of its thermal breadth, an increase in environmental temperature could drive it towards a limitation by C. On the other hand, the same temperature increase could lead to $\mathrm{P}$ limitation at the 
higher latitudinal border where the species is at the "colder" part of its thermal breadth.

In the model, the U-shape of the $T E R_{C: P}$ is strongly influenced by the shape of the $I_{c}$ thermal reaction norm. A wide $I_{c}$ thermal reaction norm breadth (thermal generalist) will lead to a "flat" $T E R_{C: P}$ U-shape. From this point on, one can explore possible links between thermal and stoichiometric traits. For example, thermal biology tells us that in some cases the wide thermal breadth of a trait (here, $I_{c}$ ) could trade-off with a low maximum performance (i.e. specialist-generalist trade-off (Angilletta, 2006)). All else kept equal in model, a low maximum $I_{c}$ implies a low maximum consumer growth rate. On the other hand, reaching a high $I_{c}$ peak performance and consequently a higher maximum growth rate could come at the cost of a narrow thermal performance breadth (thermal specialist) and a strongly convex $T E R_{C: P}$ reaching lower values (Fig. 4). Hence, the higher growth rate potential of a thermal specialist (reached at the TMT) has also a lower $T E R_{C: P}$ than that of the generalist. Biologically, this means that the specialist may require relatively more $\mathrm{P}$ to reach its full growth potential as compared with the thermal generalist. It is interesting to note how this prediction connecting high growth rate potential to higher $\mathrm{P}$ demand resembles that of the growth rate hypothesis (GRH-(Elser et al., 2003)). The GRH is based on the observation that taxa with high growth rate potential have lower body C:P ratios. Here, we show that this positive relationship between growth rate potential and $\mathrm{P}$ requirements persists even when assuming a constant body $\mathrm{C}: \mathrm{P}$ ratio. Another common pattern found in thermal biology is the "hotter is better" continuum (Angilletta et al., 2010)) in which warm-acclimated organisms reach higher maximal performances (here $I_{c}$ ) than cold-acclimated ones. Applying this into our $T E R_{C: P}$ model also yields a prediction that reminds the GRH: warm-acclimated organisms achieve higher peak $I_{c}$ and growth rates at lower $T E R_{C: P}$ than cold-adapted ones (Fig. 4).

Another influential model component is $A E_{C}$. A low $A E_{C}$ shifts the $T E R_{C: P}$ towards higher C:P ratios and increases the convexity of the U-shaped response to temperature. Biologically, $A E_{C}$ reflects the digestibility of ingested C. Detritivores or
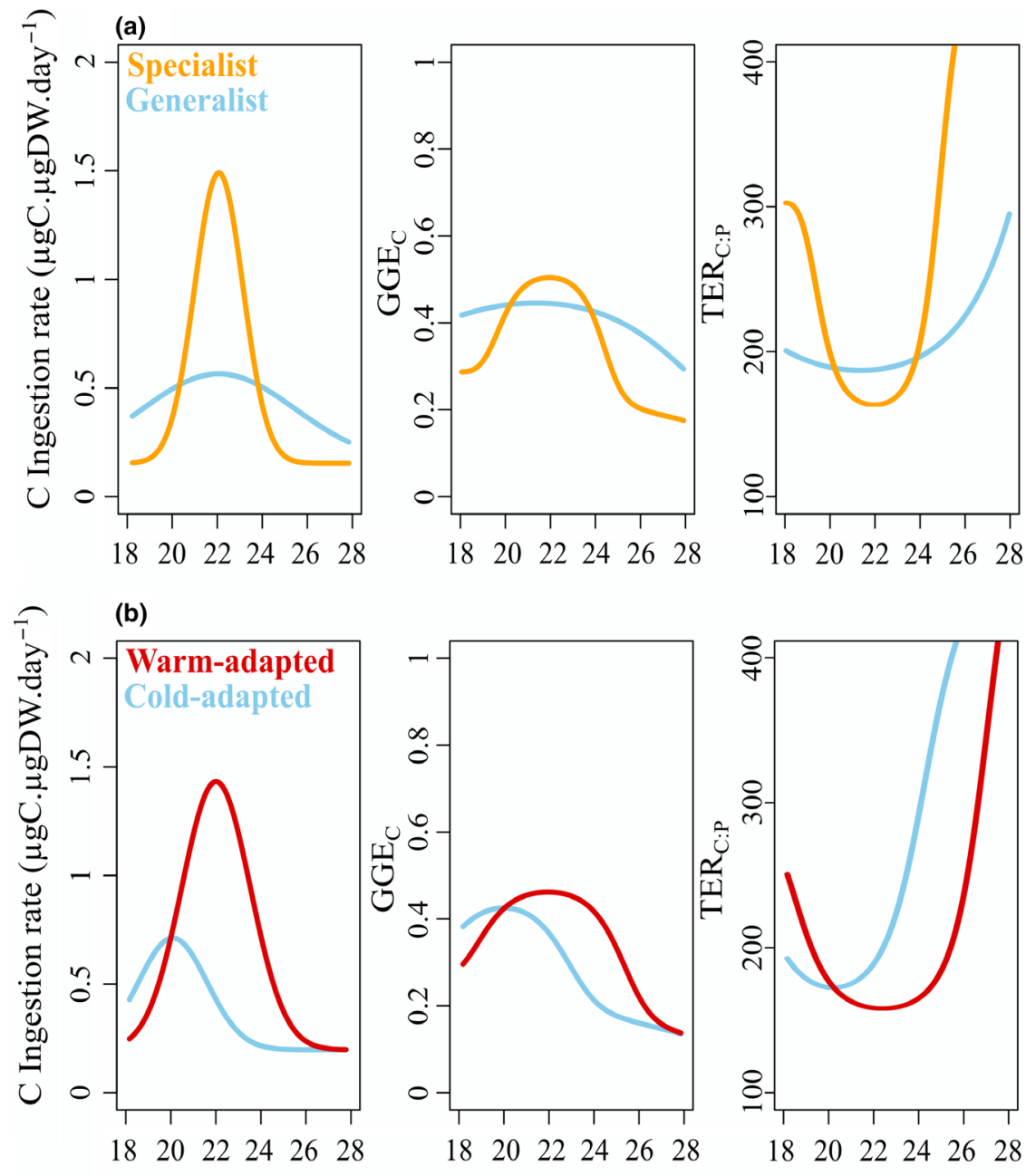

Temperature $\left({ }^{\circ} \mathrm{C}\right)$

Figure 4 Comparison of hypothetical $G G E_{C}$ and $T E R_{C: P}$ thermal reaction norms of: (a) thermal specialist (orange lines) versus thermal generalist (blue lines) consumers and (b) cold-acclimated (blue line) versus warm-acclimated (red line) consumers. Changes are induced by modifying parameters $b \Delta I_{C M A X}$ and $T_{O P T}$ (see Table 1) while keeping all other parameters constant at the values for D. magna. See text for discussion. 
terrestrial herbivores feeding on diets rich in refractory carbon should have lower $A E_{C}$ than aquatic herbivores consuming microalgae. Hence, when considering only $A E_{C}$, it is tempting to speculate that detritivores and terrestrial herbivores might be more susceptible to thermally induced dietary preference shifts or dietary-induced behavioural thermoregulation than aquatic herbivores because of their more convex $T E R_{C: P} \mathrm{U}$-shape. However, further studies are necessary to determine how the other model components vary among feeding guilds of terrestrial and aquatic ectotherms and whether they can compensate predicted effects of $A E_{C}$ on the $T E R_{C: P} \mathrm{U}$-shape.

In conclusion, we demonstrate that in D. magna the $T E R_{C}$ : $P$ responds to temperature in a U-shaped fashion. Our model simulations suggest that such a U-shaped reaction norm might be common among ectotherm consumers thereby offering a potential common explanation to previous contradictive results indicating either increases or decreases in consumer $\mathrm{P}$ demand at higher temperatures. However, the shape of thermal responses can be highly variable among traits and species (Dell et al., 2011) and the generality of the U-shaped TER remains to be tested for other species having different thermal response shapes and positions than the one provided in Table 1. Caution is also required when transposing our finding to other nutrients. The present formulation of $T E R_{C: P}$ assumes negligible P metabolic losses (Frost et al., 2006). This does not hold for $\mathrm{N}$ for which nitrogenous metabolic waste excretion represents an important fraction of the nutrient budget. Temperature accelerates metabolism and $\mathrm{N}$ excretion with unknown consequences on the $T E R_{C: N}$ thermal reaction norm (Anderson et al., 2017). Finally, body size will also differentially affect the different model components and thus potentially the shape of the $T E R_{C: P}$ response. Including the allometric size scaling in the model is clearly a promising perspective. Despite these potential caveats, our study unveils novel potential links between variation in thermal and stoichiometric traits and contributes to the on-going unification of thermal ecology and ecological stoichiometry (Hillebrand et al., 2009; Cross et al., 2015; Rosenblatt \& Schmitz, 2016).

\section{ACKNOWLEDGMENTS}

Research project "PASSCAL" funded by I-site Clermont under the "Emergence" initiative for the CAP 20-25 (awarded to $\mathrm{AB}$ ). We thank the four anonymous reviewers who provided highly constructive comments on earlier versions of this manuscript.

\section{AUTHOR CONTRIBUTIONS}

$\mathrm{AB}, \mathrm{AMK}, \mathrm{MD}$ and $\mathrm{TR}$ conceived the idea and developed the experimental protocol. TR ran the experiment and acquired the data. AMK and TR developed the model and statistical analysis. TR, AB and AMK led the writing of the manuscript. All authors contributed critically to the draft and gave the final approval for publication.

\section{COMPETING INTERESTS}

Authors declare no competing interests.

\section{DATA AND MATERIALS AVAILABILITY}

The data supporting the paper conclusions and R scripts associated are available on Figshare.

\section{REFERENCES}

Anderson, T.R. \& Hessen, D.O. (2005). Threshold elemental ratios for carbon versus phosphorus limitation in Daphnia. Freshw. Biol., 50, 2063-2075. https://doi.org/10.1111/j.1365-2427.2005.01450.x.

Anderson, T.R., Hessen, D.O., Boersma, M., Urabe, J. \& Mayor, D.J. (2017). Will invertebrates require increasingly carbon-rich food in a warming world? Am. Nat., 190, 725-742. https://doi.org/10.1086/694122.

Angilletta, M.J. (2006). Estimating and comparing thermal performance curves. J. Thermal Biol., 31, 541-545. https://doi.org/10.1016/j.jtherbio. 2006.06.002.

Angilletta, M.J., Huey, R.B. \& Frazier, M.R. (2010). Thermodynamic effects on organismal performance: is hotter better? Physiol. Biochem. Zool., 83, 197-206. https://doi.org/10.1086/648567.

Bobka, M.S., Jaeger, R.G. \& McNaught, D.C. (1981). Temperature dependent assimilation efficiencies of two species of terrestrial salamanders. Copeia, 1981, 417. https://doi.org/10.2307/1444232.

Boersma, M., Mathew, K.A., Niehoff, B., Schoo, K.L., Franco-Santos, R.M., \& Meunier, C.L. (2016), Temperature-driven changes in the diet preference of omnivorous copepods: no more meat when it's hot? A response to Winder, et al. Ecol. Lett., 19, 1386-1388. https://doi.org/10. 1111/ele.12666.

Brown, J.H., Gillooly, J.F., Allen, A.P., Savage, V.M. \& West, G.B. (2004). Toward a metabolic theory of ecology. Ecology, 85, 1771-1789.

Burton, T., Killen, S.S., Armstrong, J.D. \& Metcalfe, N.B. (2011). What causes intraspecific variation in resting metabolic rate and what are its ecological consequences? Proc. Biol. Sci., 278, 3465-3473. https://doi. org/10.1098/rspb.2011.1778.

Cherif, M. \& Loreau, M. (2013). Plant-herbivore-decomposer stoichiometric mismatches and nutrient cycling in ecosystems. Proc. Biol. Sci., 280, 20122453-20122453. https://doi.org/10.1098/rspb.2012.2453.

Clissold, F.J., Coggan, N. \& Simpson, S.J. (2013). Insect herbivores can choose microclimates to achieve nutritional homeostasis. J. Exp. Biol., 216, 2089-2096. https://doi.org/10.1242/jeb.078782.

Coggan, N., Clissold, F.J. \& Simpson, S.J. (2011). Locusts use dynamic thermoregulatory behaviour to optimize nutritional outcomes. Proc. Biol. Sci., 278, 2745-2752. https://doi.org/10.1098/rspb.2010.2675.

Cross, W.F., Hood, J.M., Benstead, J.P., Huryn, A.D. \& Nelson, D. (2015). Interactions between temperature and nutrients across levels of ecological organization. Global Change Biol., 21, 1025-1040. https://doi. org/10.1111/gcb.12809.

Darchambeau, F., Faerovig, P.J. \& Hessen, D.O. (2003). How Daphnia copes with excess carbon in its food. Oecologia, 136, 336-346. https:// doi.org/10.1007/s00442-003-1283-7.

Dell, A.I., Pawar, S. \& Savage, V.M. (2011). Systematic variation in the temperature dependence of physiological and ecological traits. Proc. Natl Acad. Sci., 108, 10591-10596. https://doi.org/10.1073/pnas.1015178108.

Denoux, C., Martin-Creuzburg, D., Koussoroplis, A.-M., Perriere, F., Desvillettes, C., Bourdier, G. et al. (2017). Phospholipid-bound eicosapentaenoic acid (EPA) supports higher fecundity than free EPA in Daphnia magna. J. Plankton Res., 39, 843-848. https://doi.org/10. 1093/plankt/fbx037.

Efron, B. \& Tibshirani, R. (1986). Bootstrap methods for standard errors, confidence intervals, and other measures of statistical accuracy. Stat. Sci., 1, 54-75. https://doi.org/10.1214/ss/1177013815.

Elser, J.J., Fagan, W.F., Denno, R. F., Dobberfuhl, D.R., Folarin, A., Huberty, A. et al. (2000). Nutritional constraint in terrestrial and freshwater food webs. Nature, 408, 578-580. https://doi.org/10.1038/ 35046058.

Elser, J.J., Acharya, K., Kyle, M., Cotner, J., Makino, W., Markow, T. et al. (2003). Growth rate-stoichiometry couplings in diverse biota. Ecol. Lett., 6, 936-943. https://doi.org/10.1046/j.1461-0248.2003.00518.x. 
Elser, J., Kyle, M., Learned, J., McCrackin, M., Peace, A. \& Steger, L. (2016). Life on the stoichiometric knife-edge: effects of high and low food C: P ratio on growth, feeding, and respiration in three Daphnia species. Inland Waters, 6, 136-146. https://doi.org/10.5268/IW-6.2.908.

Falkowski, P., Scholes, R.J., Boyle, E., Canadell, J., Canfield, D., Elser, J. et al. (2000). The global carbon cycle: a test of our knowledge of earth as a system. Science, 290, 291-296.

Frazier, M.R., Huey, R.B. \& Berrigan, D. (2006). Thermodynamics constrains the evolution of insect population growth rates: "warmer is better". Am. Nat., 168, 512-520.

Frost, P.C., Benstead, J.P., Cross, W.F., Hillebrand, H., Larson, J.H., Xenopoulos, M.A. et al. (2006). Threshold elemental ratios of carbon and phosphorus in aquatic consumers. Ecol. Lett., 9, 774-779. https:// doi.org/10.1111/j.1461-0248.2006.00919.x.

Hillebrand, H., Borer, E.T., Bracken, M.E.S., Cardinale, B.J., Cebrian, J., Cleland, E.E. et al. (2009). Herbivore metabolism and stoichiometry each constrain herbivory at different organizational scales across ecosystems. Ecology Letters, 12, 516-527. https://doi.org/10.1111/j.14610248.2009.01304.x.

Kibby, H.V. (1971). Effect of temperature on the feeding behavior of daphnia rosea. Limnol. Oceanogr., 16, 580-581. https://doi.org/10.4319/ lo.1971.16.3.0580.

Kingsolver, J.G. \& Buckley, L.B. (2017). Quantifying thermal extremes and biological variation to predict evolutionary responses to changing climate. Phil. Trans. R. Soc. B, 372, 20160147. https://doi.org/10.1098/ rstb.2016.0147.

Kingsolver, J.G., Shlichta, J.G., Ragland, G.J. \& Massie, K.R. (2006). Thermal reaction norms for caterpillar growth depend on diet. Evol. Ecol. Res., 8, 703-715.

Kreyling, J., Schweiger, A.H., Bahn, M., Ineson, P., Migliavacca, M., MorelJournel, T. et al. (2018). To replicate, or not to replicate - that is the question: how to tackle nonlinear responses in ecological experiments. Ecol. Lett., 21, 1629-1638. https://doi.org/10.1111/ele.13134.

Lampert, W. \& Trubetskova, I. (1996). Juvenile growth rate as a measure of fitness in daphnia. Func. Ecol., 10, 631. https://doi.org/10.2307/ 2390173.

Lee, K.P., Jang, T., Ravzanaadii, N. \& Rho, M.S. (2015). Macronutrient balance modulates the temperature-size rule in an ectotherm. Am. Nat., 186, 212-222.

Lemoine, N.P. \& Burkepile, D.E. (2012). Temperature-induced mismatches between consumption and metabolism reduce consumer fitness. Ecology, 93, 2483-2489.

Lemoine, N.P., Drews, W.A., Burkepile, D.E. \& Parker, J.D. (2013). Increased temperature alters feeding behavior of a generalist herbivore. Oikos, 122, 1669-1678.

Malzahn, A.M., Doerfler, D. \& Boersma, M. (2016). Junk food gets healthier when it's warm: temperature and food quality interactions on A. Tonsa. Limnol. Oceanogr., 61, 1677-1685. https://doi.org/10.1002/ $\operatorname{lno} 10330$.

Mathews, L., Faithfull, C.L., Lenz, P.H. \& Nelson, C.E. (2018). The effects of food stoichiometry and temperature on copepods are mediated by ontogeny. Oecologia, 188, 75-84. https://doi.org/10.1007/ s00442-018-4183-6.

McFeeters, B.J. \& Frost, P.C. (2011). Temperature and the effects of elemental food quality on Daphnia. Freshw. Biol., 56, 1447-1455. https://doi.org/10.1111/j.1365-2427.2011.02586.x

McMahon, J.W. (1969). Some physical factors influencing the feeding behavior of daphnia magna straus. Can. J. Zool., 43, 603-611.

Naylor, C., Bradley, M.X. \& Calow, P. (1993). Freeze-Dried chlorella vulgaris as food for daphnia magna Straus in toxicity testing. Ecotoxicol. Environ. Saf., 25, 166-172.

O'Reilly, C.M., Sharma, S., Gray, D.K., Hampton, S.E., Read, J.S., Rowley, R.J. et al. (2015). Rapid and highly variable warming of lake surface waters around the globe: global lake surface warming. Geophys. Res. Lett., 42, 10773-10781. https://doi.org/10.1002/2015GL066235.
Persson, J., Wojewodzic, M.W., Hessen, D.O. \& Andersen, T. (2011). Increased risk of phosphorus limitation at higher temperatures for Daphnia magna. Oecologia, 165, 123-129. https://doi.org/10.1007/ s00442-010-1756-4.

Prater, C., Wagner, N.D. \& Frost, P.C. (2018). Seasonal effects of food quality and temperature on body stoichiometry, biochemistry, and biomass production in Daphnia populations: Diet and temperature effects on Daphnia. Limnol. Oceanogr., 63, 1727-1740. https://doi.org/ 10.1002/lno.10803.

Rogelj, J., Meinshausen, M. \& Knutti, R. (2012). Global warming under old and new scenarios using IPCC climate sensitivity range estimates. Nat. Clim. Change, 2, 248-253. https://doi.org/10.1038/nclimate1385.

Rosenblatt, A.E. \& Schmitz, O.J. (2016). Climate change, nutrition, and bottom-up and top-down food web processes. Trends Ecol. Evol., 31, 965-975. https://doi.org/10.1016/j.tree.2016.09.009.

Ruiz, T., Bec, A., Danger, M., Koussoroplis, A.-M., Aguer, J.-P., Morel, J.-P. et al. (2018). A microcalorimetric approach for investigating stoichiometric constraints on the standard metabolic rate of a small invertebrate. Ecol. Lett., 21, 1714-1722. https://doi.org/10.1111/ele. 13137.

Schmitz, O.J. \& Rosenblatt, A.E. (2017). The temperature dependence of predation stress and prey nutritional stoichiometry. Front. Ecol. Evol., 5, 73. https://doi.org/10.3389/fevo.2017.00073.

Simonsohn, U. (2018). Two lines: A valid alternative to the invalid testing of U-shaped relationships with quadratic regressions. Advan. Methods Prac. Psychol. Sci., 1, 538-555. https://doi.org/10.1177/ 2515245918805755.

Tsai, T.-S., Lee, H.-J. \& Tu, M.-C. (2008). Specific dynamic action, apparent assimilation efficiency, and digestive rate in an arboreal pitviper, Trimeresurus stejnegeri stejnegeri. Can. J. Zool., 86, 1139-1151. https://doi.org/10.1139/Z08-090.

Urabe, J. \& Watanabe, Y. (1991). Effect of food concentration on the assimilation and production efficiencies of daphnia galeata G.O. Sars (Crustacea: Cladocera). Funct. Ecol., 5, 635. https://doi.org/10.2307/ 2389482.

Winder, M., Burian, A., Landry, M.R., Montagnes, D.J., Nielsen, J.M. (2016) Technical comment on Boersma, et al. (2016). Temperature driven changes in the diet preference of omnivorous copepods: no more meat when it's hot? Ecology Letters, 19, 45-53. Ecol. Lett., 19, 13891391. https://doi.org/10.1111/ele.12668.

Wojewodzic, M.W., Kyle, M., Elser, J.J., Hessen, D.O. \& Andersen, T. (2011). Joint effect of phosphorus limitation and temperature on alkaline phosphatase activity and somatic growth in Daphnia magna. Oecologia, 165, 837-846. https://doi.org/10.1007/s00442-010-1863-2.

Wood, S.N. (2001). mgcv: GAMs and generalized ridge regression for R. $R$ news, 1, 20-25.

Yurista, P.M. (1999). Temperature-dependent energy budget of an Arctic Cladoceran, Daphnia middendorffiana: D. middendorffianae. Freshw. Biol., 42, 21-34. https://doi.org/10.1046/j.1365-2427.1999.00446.x.

Zhou, L., Declerck, S.A.J. (2019). Herbivore consumers face different challenges along opposite sides of the stoichiometric knife-edge. Ecol. Lett., 22, 2018-2027. https://doi.org/10.1111/ele.13386.

\section{SUPPORTING INFORMATION}

Additional supporting information may be found online in the Supporting Information section at the end of the article.

Editor, Ian Donohue

Manuscript received 2 January 2020

First decision made 13 February 2020

Manuscript accepted 22 February 2020 